\title{
Comparison of Relativistic Iron Line Models
}

\author{
Jiří Svoboda, ${ }^{a b}$ Michal Dovčiak, ${ }^{a}$ René W. Goosmann ${ }^{a c}$ and Vladimír Karas ${ }^{a}$ \\ ${ }^{a}$ Astronomical Institute of the Academy of Sciences, Prague, Czech Republic \\ ${ }^{b}$ Charles University, Faculty of Mathematics and Physics, Prague, Czech Republic \\ ${ }^{c}$ Observatoire Astronomique de Strasbourg, Strasbourg, France \\ E-mail: jirik.svoboda@gmail.com,dovciak@astro.cas.cz, \\ goosmanneastro.cas.cz, vladimir.karas@cuni.cz
}

\begin{abstract}
The analysis of the broad iron line profile in the X-ray spectra of active galactic nuclei and black hole X-ray binaries allows us to constrain the spin parameter of the black hole. We compare the constraints on the spin value for two X-ray sources, MCG-6-30-15 and GX 339-4, with a broad iron line using present relativistic line models in XSPEC — LAOR and KYRLINE. The LAOR model has the spin value set to the extremal value $a=0.9982$, while the KYRLINE model enables direct fitting of the spin parameter. The spin value is constrained mainly by the lower boundary of the broad line, which depends on the inner boundary of the disk emission where the gravitational redshift is maximal. The position of the inner disk boundary is usually identified with the marginally stable orbit which is related to the spin value. In this way the LAOR model can be used to estimate the spin value. We investigate the consistency of the LAOR and KYRLINE models. We find that the LAOR model tends to overestimate the spin value. However, the discrepancies between results of both models are within the general uncertainties when applied on the current data.
\end{abstract}

VII Microquasar Workshop: Microquasars and Beyond

September 1-5 2008

Foca, Izmir, Turkey 


\section{Introduction}

The broad emission iron lines are well-known features found in about two dozens of spectra of active galactic nuclei and black hole binaries. They are supposed to originate close to the black hole by the reflection of the primary radiation on the accretion disk. The spin of the black hole plays an important role in the forming of the line shape. Especially, it determines the position of the marginally stable orbit which is supposed to confine the inner edge of the accretion disk.

$$
r_{\mathrm{ms}}=3+Z_{2}-\left[\left(3-Z_{1}\right)\left(3+Z_{1}+2 Z_{2}\right)\right]^{\frac{1}{2}},
$$

where $Z_{1}=1+\left(1-a^{2}\right)^{\frac{1}{3}}\left[(1+a)^{\frac{1}{3}}+(1-a)^{\frac{1}{3}}\right]$ and $Z_{2}=\left(3 a^{2}+Z_{1}^{2}\right)^{\frac{1}{2}}$. The innermost stable orbit occurs closer to a black hole with a higher spin value. However, the spin affects also the overall shape of the line.

Over almost two decades the most widely used model of the relativistic disk spectral line has been the one by Laor (1991), which includes the effects of a maximally rotating Kerr black hole. In other words, the LAOR model sets the dimensionless angular momentum $a$ to the canonical value of $a=0.9982$ - so that it cannot be subject of the data fitting procedure. Dovčiak et al. (2004) have relaxed this limitation and allowed $a$ to be fitted in the suite of KY models. Other numerical codes have been developed independently by several groups (Beckwith \& Done, 2004; Čadež \& Calvani, 2005; Brenneman \& Reynolds, 2006) and equipped with similar functionality.

However, the LAOR model can still be used for evaluation of the spin if one identifies the inner edge of the disk with the marginally stable orbit. In this case the spin is actually estimated from the lower boundary of the broad line. The other parameters of the relativistic line models are inclination angle $i$, rest energy of the line $E$, inner radius of the disk $R_{\text {in }}$, outer radius of the disk $R_{\text {out }}$, emissivity parameters $q_{1}, q_{2}$ with the break radius $r_{b}$. The emissivity of the line is given by $I \approx r^{-q_{1}}$ for $r<r_{b}$ and $I \approx r^{-q_{2}}$ for $r>r_{b}$. The angular dependence of the emissivity is characterized by limb darkening profile $I\left(\mu_{\mathrm{e}}\right) \propto 1+2.06 \mu_{\mathrm{e}}$ in the LAOR model. The KYRLINE model enables to switch between different emission laws. We used further two extreme cases, the KYRLINE with the same limb-darkening law as in the LAOR model and KYRLINE* with the limb-brightening law $I\left(\mu_{\mathrm{e}}\right) \propto \ln \left(1+\frac{1}{\cos i}\right)$.

The aim of this paper is to compare the two models applied to the current data provided by the XMM-Newton satellite. For this purpose we have chosen two sources, MCG-6-30-15 and GX 3394, which exhibit an extremely skewed iron line according to recently published papers (Vaughan \& Fabian, 2004; Miller et al., 2004).

\section{Observations and data reduction}

We used the SAS software version 7.1.2 (http://xmm.esac.esa.int/sas) to reduce the XMMNewton data of the sources. Further, we used standard tools for preparing and fitting the data available at http://heasarc.gsfc.nasa.gov (FTOOLS, XSPEC)

The galaxy MCG-6-30-15 is a nearby Seyfert 1 galaxy $(z=0.008)$. The skewed iron line has been revealed in the X-ray spectra by all recent satellites. The XMM-Newton observed MCG-6-3015 for a long $350 \mathrm{ks}$ exposure time during summer 2001 (revolutions 301, 302, 303). The spectral 
results are described in Fabian et al. (2002). We joined the three spectra into one using the ftool MATHPHA.

The black hole binary GX 339-4 exhibited a strong broadened line in the 76 ks observation in 2002 (Miller et al., 2004) when the source was in the very high state. The observation was made in the burst mode due to a very high source flux. The $97 \%$ of photons are lost during the reading cycle in this mode, which results into $2.25 \mathrm{ks}$ total exposure time. ${ }^{1}$

We rebinned all the data channels in order to oversample the instrumental energy resolution maximally by a factor of 3 and to have at least 20 counts per bin. The first condition is much stronger with respect to the total flux of the sources $-4 \times 10^{-11} \mathrm{erg} \mathrm{cm}^{-2} \mathrm{~s}^{-1}$ in $2-10 \mathrm{keV}(1.1 \times$ $\left.10^{6} \mathrm{cts}\right)$ for MCG-6-30-15 and $9 \times 10^{-9} \mathrm{erg} \mathrm{cm}^{-2} \mathrm{~s}^{-1}$ in $2-10 \mathrm{keV}\left(1.0 \times 10^{7} \mathrm{cts}\right)$ for GX 339-4.

\section{Iron line study of the MCG-6-30-15 spectrum}
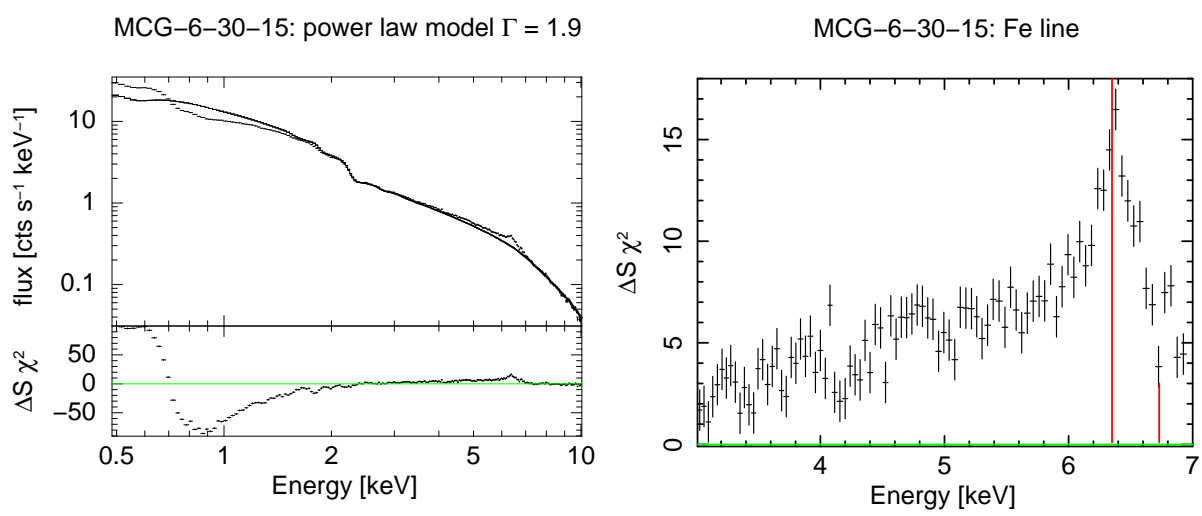

Figure 1: The X-ray spectrum of MCG-6-30-15 observed by XMM-Newton. Left: The overall view on the spectrum as a power law with $\Gamma=1.9$ absorbed by a neutral hydrogen along the line of sight with column density $n_{\mathrm{H}}=0.41 \times 10^{21} \mathrm{~cm}^{-2}$. The residuals from the model are plotted in the bottom panel clearly revealing features of a local absorption and soft excess at the soft X-ray band, and a feature at around $6 \mathrm{keV}$ which can be explained by the presence of a broad iron line. Right: More detailed view of the iron line band.

We used the same continuum model for the MCG-6-30-15 spectrum as presented in Fabian et al. (2002): the simple power law component absorbed by neutral hydrogen with the column density $n_{\mathrm{H}}=0.41 \times 10^{21} \mathrm{~cm}^{-2}$. The overall spectrum with a detailed view of the iron line energy band is shown in Figure 1. The employed model is sufficient to fit the data above $\approx 2.5 \mathrm{keV}$, which is satisfactory for our goal of the comparison of the relativistic line models. ${ }^{2}$ The value

\footnotetext{
${ }^{1}$ The broad iron line was also revealed in the analysis of the two $138 \mathrm{ks}$ observations in spring 2004 by Miller et al. (2006), when the source was in the low-hard state. The EPIC pn camera was operating in the timing mode, MOS cameras in the full-frame mode. Using epatplot tool we found that it is not possible to avoid the pile-up by excluding the central part of the image of the source as described in the forementioned paper. Therefore we use only the very high state observation from 2002 in our analysis.

${ }^{2}$ Another components are needed to be added into the model in order to fully understand the spectrum. Several works have been done in this way, in the most recent one by Miller et al. (2008) the spectrum is characterized by an absorption in four different zones, which affects also the higher energy band where no broad line is needed any more.
} 
of the photon index is $\Gamma=1.90(1)$. The residuals are formed by a complex of a broad iron line and two narrow iron lines - one emission line at $E=6.4 \mathrm{keV}$ likely originating in a distant matter (torus) and one absorption line at $E=6.77 \mathrm{keV}$ which can be explained by a blueshifted absorption originating in an outflow. The rest energy of the broad line is $E=6.7 \mathrm{keV}$, which corresponds to the helium-like ionized iron atoms. ${ }^{3}$

A good fit of the broad line was found with a broken power law line emissivity with a steeper dependence on the radius in the innermost region, which suggests a centrally localized corona. The goodness of the fit is constrained by the least squared method. The fit results in $2.5-9.5 \mathrm{keV}$ are presented in Table 1. The $\chi^{2}$ values give comparable results for all employed models. The $\chi_{\text {red }}^{2}=\chi^{2} / v \approx 1.2$, where $v$ is the number of degrees of freedom which is related to the total number of energy bins and model parameters. The six independent parameters of the LAOR and KYRLINE models make the global minimum of $\chi^{2}$ rather wide with several local minima. Each model has a different tendency to converge to a different minimum. Hence, we did not compare only best fits of both models, but also the evaluated spin values by the KYRLINE and LAOR models when the other model parameters correspond to each other. The equivalent width of the line is $E W \approx 750 \mathrm{eV}$. The errors in brackets presented in the table correspond to $90 \%$ confidence and are evaluated when the other parameters of the model are fixed. The realistic errors are higher because the model parameters further depend on the other parameters of the line and continuum models.

To catch up these relations we produce various contour graphs focusing on the determination of the spin value, taking into account the other parameters of the used model. The relations of the $\chi^{2}$ values on value of the spin (KYRLINE) or the inner disk radius (LAOR) are shown in the left column of Figure 2. The x-axis is oppositely directed in the case of the inner disk radius as $\mathrm{x}$-variable for an easier comparison with the KYRLINE results. The contour graphs for the spin and the inclination angle are shown in the middle column of Figure 2. The underlying model was fixed in both cases. The plots in the right column of Figure 2 show the contours for the spin and the power law index. Taking all of these into account, we obtain for the spin value:

$$
a_{K Y}=0.94_{-0.10}^{+0.06} \text { and } \quad a_{\text {laor }}=0.96_{-0.08}^{+0.04}
$$

\section{Iron line study of the GX 339-4 spectrum}

The continuum of the X-ray spectrum of the black hole binaries is characterized by a power law and a multicolor disk black-body component (POWERLAW + DISKBB in the XSPEC notation). The power law index suggested from the simultaneous RXTE measurements is $\Gamma=2.5$ (Miller et al., 2004). However, we get an unacceptable fit with $\chi_{\text {reduced }}^{2} \geq 6.7$ using the same model as in the analysis by Miller et al. (2004) or the re-analysis by Reis et al. (2008). The difference of the results is likely due to a different grouping of the instrumental energy channels applied to the data. While we did not allow to oversample the instrumental energy resolution more than by factor of 3 , in the previous works only the condition to have at least 20 counts per bin was used. This

\footnotetext{
${ }^{3}$ The spectral complexity in the line band allows an alternative explanation - the model with two narrow emission lines at energies $E=6.4 \mathrm{keV}$ and $E=6.97 \mathrm{keV}$. This alternative model leads to the presence of the broad line component at $E=6.4 \mathrm{keV}$.
} 


\begin{tabular}{c|c|c|c|c}
\hline \hline parameter & KYRLINE & KYRLINE* & LAOR best & LAOR loc.min. $^{+0.02}$ \\
\hline$a / M$ & $\mathbf{0 . 9 4}_{-0.03}^{+0.02}$ & $\mathbf{0 . 9 5}_{-0.01}^{+0.02}$ & $\mathbf{0 . 9 8}_{-0.01}^{+0.02}$ & $\mathbf{0 . 9 6}_{-0.01}^{+0.02}$ \\
$i[\mathrm{deg}]$ & $26.7(7)$ & $31.5(7)$ & $35.7(5)$ & $26.8(5)$ \\
$E[\mathrm{keV}]$ & $6.67(1)$ & $6.60(1)$ & $6.48(1)$ & $6.66(1)$ \\
$q_{1}$ & $4.9(1)$ & $3.7(1)$ & $4.8(1)$ & $4.7(1)$ \\
$q_{2}$ & $2.84(4)$ & $2.11(4)$ & $2.50(3)$ & $2.87(3)$ \\
$r_{b}$ & $5.5(2)$ & $18.3(5)$ & $6.6(2)$ & $5.1(2)$ \\
\hline$\chi^{2} / v$ & $175 / 148$ & $174 / 148$ & $170 / 148$ & $174 / 148$ \\
\hline$E W[\mathrm{eV}]$ & 761 & 757 & 764 & 754
\end{tabular}

Table 1: Results for the iron line parameters for MCG-6-30-15 in 2.5-9.5 keV.
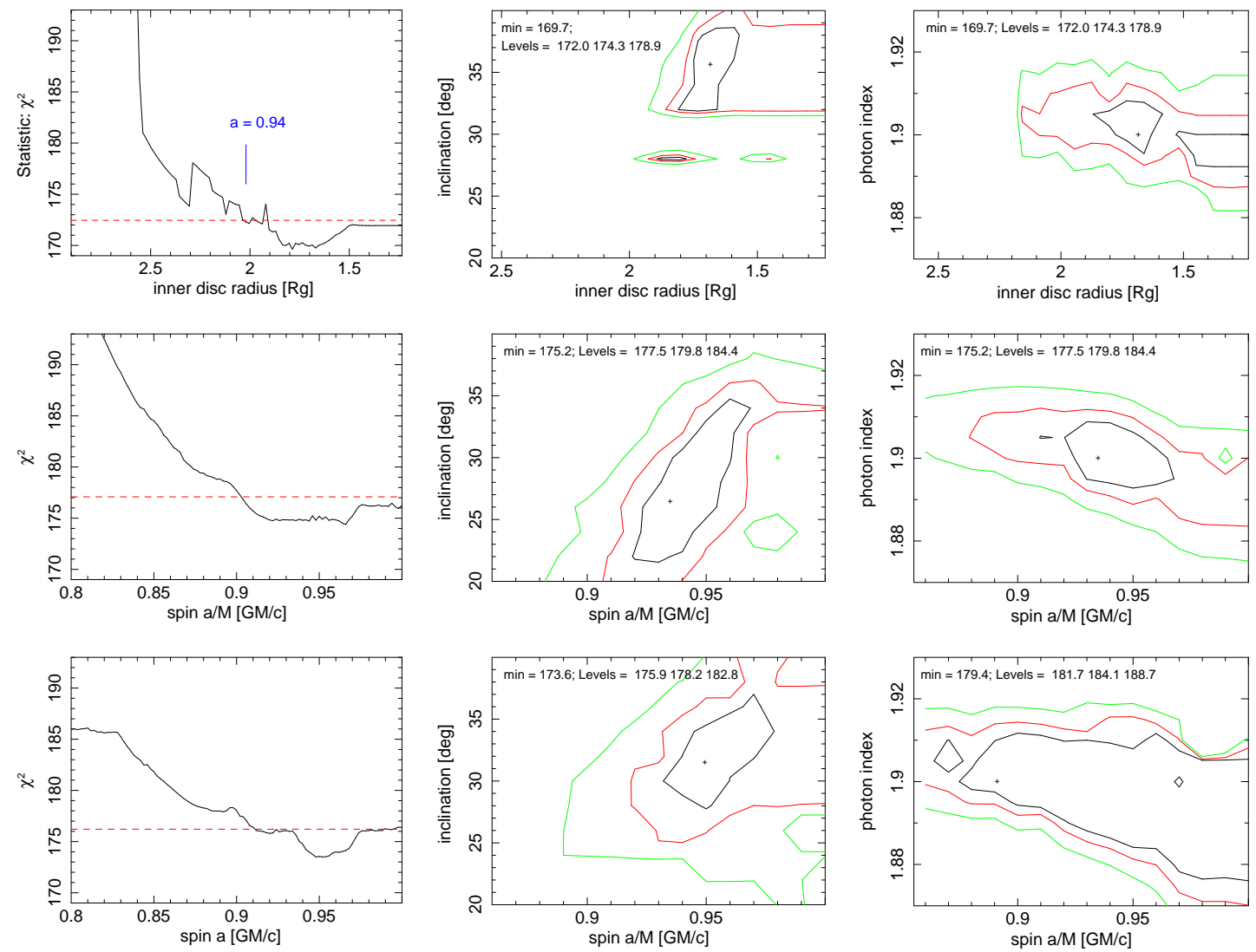

Figure 2: The contour graphs show the dependence of the value of the $\chi^{2}$, the inclination angle and the power law index on the value of the spin (KYRLINE) or the inner disk radius (LAOR) for the MCG-6-30-15 spectrum in $2.5-9.5 \mathrm{keV}$. The black, red and green contours correspond to $1 \sigma, 2 \sigma$ and $3 \sigma$, respectively. Top: The results of the LAOR model. Middle: The results of the KYRLINE model with limb darkening. Bottom: The results of the KYRLINE model with limb brightening.

condition is very weak with respect to the total number of counts $N_{\text {counts }} \approx 1.0 \times 10^{7}$ and the total number of energy channels $N_{\text {chan }}=1.5 \times 10^{3}$ in $2-10 \mathrm{keV}$ and as a result, it practically does not force the data to be grouped. This leads to an excessive oversampling of the energy resolution, to 

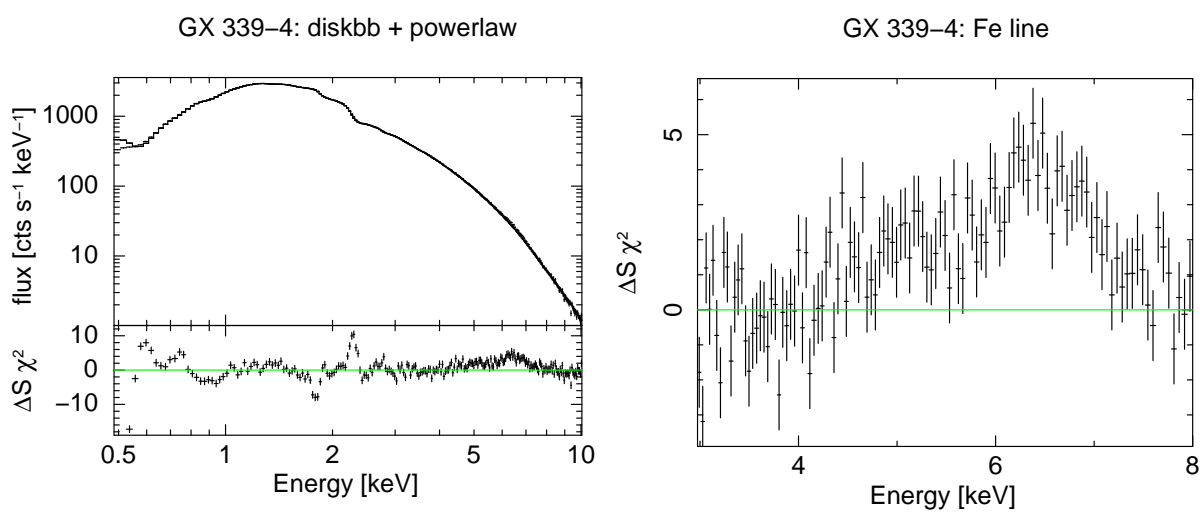

Figure 3: The X-ray spectrum of GX 339-4. Left: The overall view on the spectrum as a power law with $\Gamma=3$ and thermal multi temperature black body with $0.87 \mathrm{keV}$ absorbed by a neutral hydrogen with column density $n_{\mathrm{H}}=0.6 \times 10^{22} \mathrm{~cm}^{-2}$. The residuals from the model are plotted in the bottom panel revealing wiggles at the soft X-ray band which are likely due to instrumental response. The broad excess at around $6 \mathrm{keV}$ can be explained by the presence of a broad iron line. Right: More detailed view of the iron line band.

large error bars in the flux and finally to an artificial decrease of the $\chi_{\text {red }}^{2}$ value. In the energy range $0.8-9 \mathrm{keV}$ we get $\chi^{2} / v=2835 / 1640$ for the grouping with the only condition 20 counts per bin, and $\chi^{2} / v=1368 / 202$ for the grouping taking the energy resolution into account. The $\chi_{\text {red }}^{2}$ value increased from $\chi_{\text {red }}^{2} \doteq 1.73$ to $\chi_{\text {red }}^{2} \doteq 6.77$.

The significant effect on the goodness of the fit is due to the features at $E \approx 1.8 \mathrm{keV}$ and $E \approx 2.3 \mathrm{keV}$, which can be linked with the $\mathrm{Si}$ and Au edges suggesting to be instrumental imprints. Hence we added two gaussian lines into the model to improve the fit $\left(\chi^{2} / v \rightarrow 667 / 202\right)$. As a next step, we allowed the continuum parameters to float (and also removed the SMEDGE model used in the previous works). The new model has $\chi^{2} / v=350 / 202$ in $0.8-9 \mathrm{keV}$ and its parameter values are $n_{\mathrm{H}}=0.6 \times 10^{22} \mathrm{~cm}^{-2}, \Gamma=3.08(5)$ and $k T_{\text {in }}=0.87(1) \mathrm{keV}$. We tried to add a reflection component into the model, as PEXRAV (PEXRIV) or REFSCH model, but without any improvements of the fit. The spectrum of GX 339-4 is shown in Figure 3 with a detailed view of the iron line band in the right panel. A broadened iron line feature is still present. However, due to different adopted value for the photon index of the power law the line is much weaker than the one presented in Miller et al. (2004).

The fitting results of the line models in 3-9 keV are summarized in Table 2 and Figure 4. There are two minima found during the fitting procedure. We preferred the one which better corresponds to the results obtained by the independent radio and infrared measurements which constrained the inclination angle to be $i<26^{\circ}$ (Gallo et al., 2004). The dependence of the goodness of the fit on the spin value is shown in the left column of Figure 4. The contour graphs for the spin and the inclination angle are depicted in the middle column, and for the spin and the power law photon index in the right column of Figure 4. The derived spin value is then:

$$
a_{K Y}=0.69_{-0.13}^{+0.16} \text { and } \mathrm{a}_{\mathrm{laor}}=0.77_{-0.14}^{+0.10}
$$




\begin{tabular}{c|c|c|c}
\hline \hline parameter & KYRLINE & KYRLINE* & LAOR \\
\hline$a / M$ & $\mathbf{0 . 6 9}_{-0.12}^{+0.13}$ & $\mathbf{0 . 6 2}_{-0.14}^{+0.14}$ & $\mathbf{0 . 7 7}_{-0.12}^{+0.08}$ \\
$i[\mathrm{deg}]$ & $19(3)$ & $19(4)$ & $17(4)$ \\
$E[\mathrm{keV}]$ & $6.97(1)$ & $6.97(1)$ & $6.97(1)$ \\
$q$ & $3.45(8)$ & $3.35(8)$ & $3.3(1)$ \\
\hline$\chi^{2} / v$ & $147 / 125$ & $148 / 125$ & $148 / 125$ \\
\hline$E W[\mathrm{eV}]$ & 175 & 164 & 164
\end{tabular}

Table 2: Results for the iron line parameters for GX 339-4 in 3-9 keV
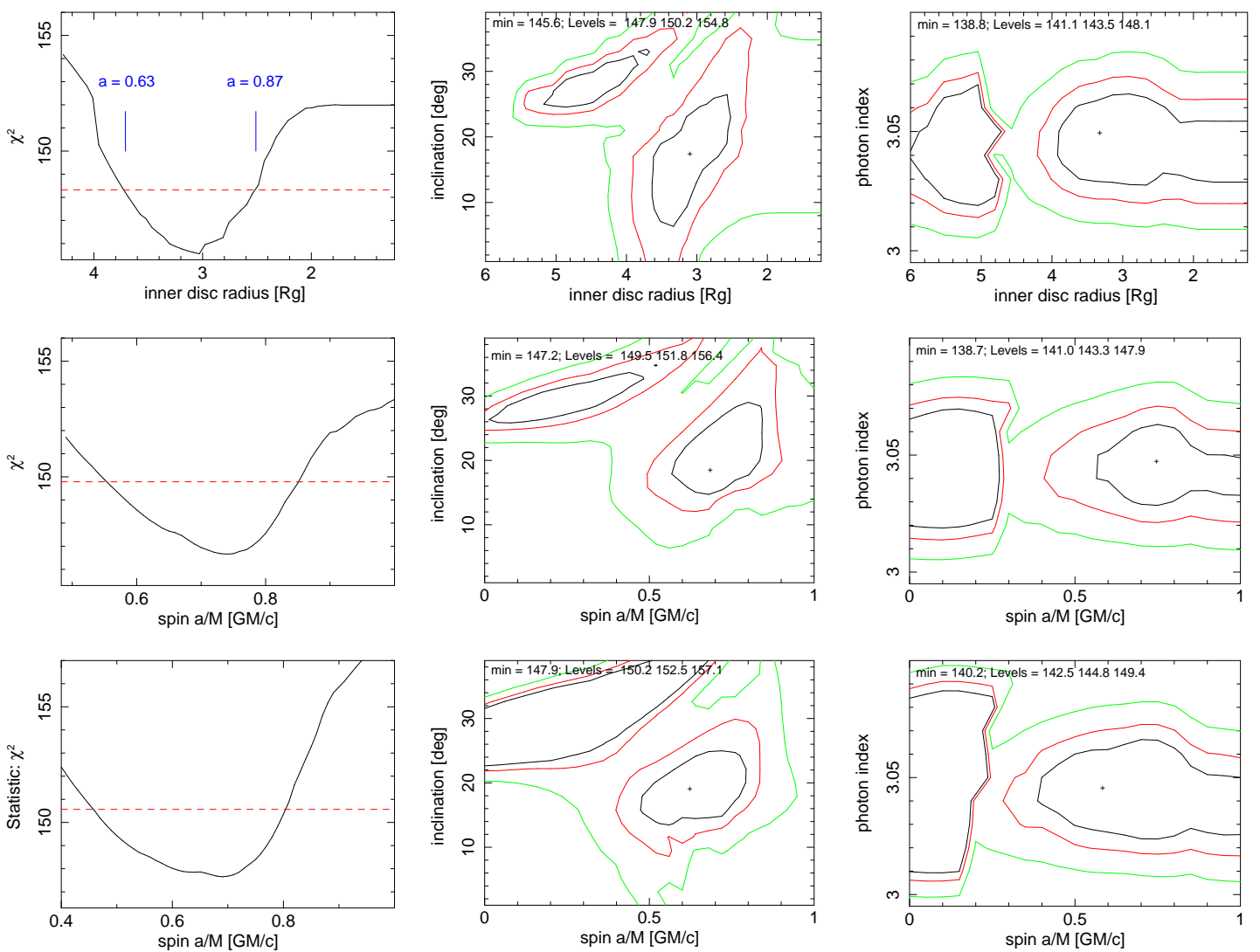

Figure 4: The contour graphs show the dependence of the value of the $\chi^{2}$, the inclination angle and the power law index on the value of the spin (KYRLINE) or the inner disk radius (LAOR) for the GX 339-4 spectrum in $3-9 \mathrm{keV}$. The black, red and green contours correspond to $1 \sigma, 2 \sigma$ and $3 \sigma$, respectively. Top: The results of the LAOR model. Middle: The results of the KYRLINE model with limb darkening. Bottom: The results of the KYRLINE model with limb brightening.

\section{Conclusions}

We investigated the iron line band for two representative sources - MCG-6-30-15 (active galaxy) and GX 339-4 (X-ray binary). The iron line is statistically better constrained for the active 


\begin{tabular}{c|c|c}
\hline \hline & MCG-6-30-15 & GX 339-4 \\
\hline net cts/s & 3.59 & 592.1 \\
model cts/s & 3.59 & 592.5 \\
line cts/s & 0.20 & 5.1 \\
line cts & $4.37 \times 10^{4}$ & $1.15 \times 10^{4}$
\end{tabular}

Table 3: Count rates of the observations.

galaxy MCG-6-30-15 due to a significantly longer exposure time of the available observations for comparison of count rates of the sources see Table 3. The spectra of both sources are well described by a continuum model plus a broad iron line model. We compared modeling of the broad iron line by the two relativistic models, LAOR and KYRLINE. The main difference between both models is in the determination of the spin value. The spin value is not fitted directly by the LAOR model. However, it can be estimated from the value of the inner radius of the disk, if we identify the inner disk boundary with the marginally stable orbit. For MCG-6-30-15 we get: $a_{K Y}=0.94_{-0.10}^{+0.06}$ and $a_{\text {laor }}=0.96_{-0.08}^{+0.04}$, for GX 339-4: $a_{K Y}=0.69_{-0.13}^{+0.16}$ and $a_{\text {laor }}=0.77_{-0.14}^{+0.10}$. Apparently, the LAOR model tends to overestimate the spin value. The discrepancies between the KYRLINE and LAOR results are however within the general uncertainties of the spin determination using the skewed line profile when applied to the current data.

The KYRLINE model leads to a better defined minimum of $\chi^{2}$ for the best fit value. The confidence contour plots for $a / M$ versus other model parameters are more regularly shaped. This indicates that the KYRLINE model has a smoother adjustment between the different points in the parameter space allowing for more reliable constraints on $a / M$, while the LAOR model has a less accurate grid.

As a side-product, we find that the correct re-binning of the data with respects to the instrumental energy resolution is crucial to obtain the most statistically relevant results. The re-binning is done insufficiently in several recent papers, however.

\section{Acknowledgments}

The present work was supported by the Charles University Grant Agency under Contract 33308 .

\section{References}

Beckwith, K. \& Done, C. 2004, MNRAS, 352, 353

Brenneman, L. W. \& Reynolds, C. S. 2006, ApJ, 652, 1028

Dovčiak, M., Karas, V., \& Yaqoob, T. 2004, ApJS, 153, 205

Fabian, A. C., Vaughan, S., Nandra, K., Iwasawa, K., Ballantyne, D. R., Lee, J. C., De Rosa, A., Turner, A., \& Young, A. J. 2002, MNRAS, 335, L1 
Gallo, E., Corbel, S., Fender, R. P., Maccarone, T. J., \& Tzioumis, A. K. 2004, MNRAS, 347, L52

Laor, A. 1991, ApJ, 376, 90

Miller, J. M., Fabian, A. C., Reynolds, C. S., Nowak, M. A., Homan, J., Freyberg, M. J., Ehle, M., Belloni, T., Wijnands, R., van der Klis, M., Charles, P. A., \& Lewin, W. H. G. 2004, ApJ, 606, L131

Miller, J. M., Homan, J., Steeghs, D., Rupen, M., Hunstead, R. W., Wijnands, R., Charles, P. A., \& Fabian, A. C. 2006, ApJ, 653, 525

Miller, L., Turner, T. J., \& Reeves, J. N. 2008, A\&A, 483, 437

Reis, R. C., Fabian, A. C., Ross, R. R., Miniutti, G., Miller, J. M., \& Reynolds, C. 2008, MNRAS, 387, 1489

Čadež, A. \& Calvani, M. 2005, MNRAS, 363, 177

Vaughan, S. \& Fabian, A. C. 2004, MNRAS, 348, 1415 